\title{
CAMBRIDGE
}

\section{Introduction to the Modern Theory of Dynamical Systems}

\section{ANATOLE KATOK and BORIS HASSELBLATT}

This book provides the first self-contained comprehensive exposition of the theory of dynamical systems as a core mathematical discipline closely intertwined with all main areas of mathematics. The authors introduce and rigorously develop the theory while providing researchers interested in applications with fundamental tools and paradigms.

f60.00 net HB $0521341876 \quad 822$ pp. 1995

Encyclopedia of Mathematics and its Applications 54

\section{Ergodic Theory and Harmonic Analysis}

Proceedings of the 1993 Alexandria Conference

Edited by KARL E. PETERSEN and IBRAHIM SALAMA

The papers printed here explore many of the rapidly developing connections between ergodic theory and other branches of mathematics, giving the background of each area, the most outstanding recent results and the most current promising lines of research. They should form perfect starting points for beginning researchers.

f24.95 net PB $0521459990 \quad 445$ pp. 1995

London Mathematical Society Lecture Note Series 205

\section{Lectures on Ergodic Theory and Pesin Theory on Compact Manifolds}

\section{MARK POLLICOTT}

Pesin theory consists of the study of the theory of non-uniformly hyperbolic diffeomorphisms. The aim of this book is to provide the reader with a straightforward account of this theory, following the approaches of Katok and Newhouse. The notes are divided into two parts. The first develops the basic theory, starting with general ergodic theory and introducing Liapunov exponents. Part Two deals with the applications of Pesin theory and contains an account of the existence (and distribution) of periodic points. It closes with a look at stable manifolds, and gives some results on absolute continuity.

$£ 19.95$ net PB $0521435935 \quad 180$ pp. 1992

London Mathematical Society Lecture Note Series 180

Cambridge books are available from good bookshops, alternatively call UK $+44(0) 1223325970$ to order direct using your credit card, or fax UK $+44(0) 1223$ 315052. For further information please email Giulia Williams on science@cup.cam.ac.uk 


\section{INSTRUCTIONS TO AUTHORS}

\section{Submission of typescripts}

Two copies of the manuscript should be submitted to one of the four Executive Editors (addresses on outside front cover). Papers may also be submitted electronically to those Executive Editors with electronic mail addresses. The editor will acknowledge receipt of the manuscripts. It is important that authors inform the editor of any changes of address whilst their paper is under consideration.

\section{Typescript}

Papers should be typed, double-spaced, on one side only and with generous margins. The pages must be numbered.

The first page should give the title, the author's name and institution, and a short abstract intelligible to mathematicians.

The title, while brief, must be informative (e.g. $A$ new proof of the ergodic theorem, whereas Some applications of a theorem of Birkhoff would be useless).

\section{Notation}

It is important that mathematicians expressions are clear to a printer (who is not a mathematician). For instance, $n_{k}(n$ sub $k$ ) is common usage, but avoid if possible using $c$ sub $n$ sub $k$. Fractions are generally best expressed by a solidus. Complicated exponents like

$$
\exp \left\{z^{2} \sin \theta /\left(1+y^{2}\right)\right\}
$$

should be shown in this and no other way.

In the typescript, italics, small capitals and capitals are specified by single, double and triple underlining. Bold-faced types is shown by wavy underlining.

It helps if displayed equations or statements which will be quoted later are numbered in order on the right of their line. They can then be referred to by, for example, 'from (7)'.

The author must enable the printer (if necessary by pencilled notes in the margin) to distinguish between similar symbols such as $o, O, \mathrm{o}, \mathrm{O}, 0 ; x$, $\mathrm{X}, \mathrm{X} ; \phi, \Phi, \varnothing ; 1,1 ; \varepsilon, \epsilon ; \kappa, k$.

There is no need to underline Greek or script letters provided these are clearly typed. Any special symbols should be explained on a separate sheet of directions for the printer.

If an author wishes to mark the end of the proof of a theorem, the sign $\square$ may be used.

Footnotes should be avoided.

\section{Diagrams}

Figures and drawings should be on separate sheets in black ink. Photocopies are acceptable only if they are as clear as the originals. Symbols, legends and captions should be given on a transparent overlay. Each text figure must be numbered as Figure 1, Figure 2, $\ldots$ and its intended position clearly indicated in the typescript. The author's name in pencil must be on all separate sheets of diagrams.

A figure is expensive to reproduce and should be included only when the subject matter demands it, or when it greatly clarifies the exposition.

The publisher recognizes that some authors do not have the facilities for producing drawings of a sufficiently high standard to be reproduced directly and is therefore willing to have such diagrams redrawn, provided that they are clear.

\section{Tables}

Tables should be numbered (above the table) and set out on separate sheets. Indicate the position of each in the text as for figures.

\section{References}

References should be collected at the end of the paper numbered in alphabetical order of the author's names. A reference to a book should give the title, in italics, and then in roman type the publisher's name and the place and year of publication;

[4] N. Dunford \& J. T. Schwartz Linear Operators Part I. Wiley: New York, 1958.

A reference to a paper should give in italics the title of the periodical, the number of the volume and year, and the beginning and end pages of the paper. Titles should be abbreviated as in Mathematical Reviews:

[6] J. E. Littlewood. The 'pits effect' for functions in the unit circle. J. Analyse Math. 23 (1970), $236-268$

\section{Proofs}

Authors receive one set of proofs for correction. If excessive alterations to the original manuscript are requested after the paper has been typeset, the author will be charged the cost of resetting. For papers with more than one author the proofs are sent to the first named author unless the editor receives other instructions. It is important that proofs are corrected and returned promptly.

\section{Reprints}

There are 100 reprints, free of charge, for each paper. For papers with several authors these reprints are divided between the authors. There are no page charges. 


\section{Ergodic theory and dynamical systems}

VOLUME 15 PART 4 AUGUST 1995

\section{CONTENTS}

F. Blanchard et al Entropy pairs for a measure

$D$. Gaboriau et al Pseudogroups of isometries of $\mathbb{R}:$ reconstruction of free actions on $\mathbb{R}$-trees

C. Kraaikamp and R. Meester Ergodic properties of a dynamical system arising from percolation theory

A. Maass On the sofic limit sets of cellular automata

W. Parry Instances of cohomological triviality and rigidity

A. A. Pinto and D. A. Rand Classifying $\mathrm{C}^{1+}$ structures on dynamical fractals: 1. The moduli space of solenoid functions for Markov maps on train tracks

N. Romero Persistence of homoclinic tangencies in higher dimensions

$K$. Schmidt Cohomological rigidity of algebraic $\mathbb{Z}^{d}$-actions

C. Yue Conditional measure and flip invariance of Bowen-Margulis and harmonic measures on manifolds of negative curvature

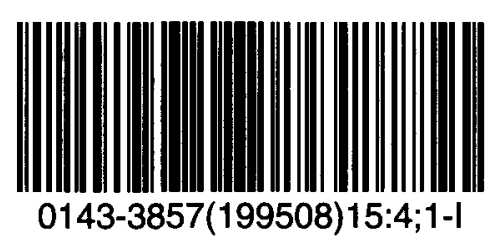

